\title{
Material Measurement Method Based on Femtosecond Laser Plasma Shock Wave
}

\author{
Dong $\mathrm{ZHONG}^{*}$ and Zhongming LI \\ School of Electronic and Information, Hubei University of Science and Technology, Xianning, 437100, China \\ *Corresponding author: Dong ZHONGＥ-mail: zhongdong0129@163.com
}

\begin{abstract}
The acoustic emission signal of laser plasma shock wave, which comes into being when femtosecond laser ablates pure $\mathrm{Cu}, \mathrm{Fe}$, and $\mathrm{Al}$ target material, has been detected by using the fiber Fabry-Perot (F-P) acoustic emission sensing probe. The spectrum characters of the acoustic emission signals for three kinds of materials have been analyzed and studied by using Fourier transform. The results show that the frequencies of the acoustic emission signals detected from the three kinds of materials are different. Meanwhile, the frequencies are almost identical for the same materials under different ablation energies and detection ranges. Certainly, the amplitudes of the spectral character of the three materials show a fixed pattern. The experimental results and methods suggest a potential application of the plasma shock wave on-line measurement based on the femtosecond laser ablating target by using the fiber F-P acoustic emission sensor probe.
\end{abstract}

Keywords: Optical fiber sensing; femtosecond laser; plasma shock wave; acoustic signal; material testing

Citation: Dong ZHONG and Zhongming LI, "Material Measurement Method Based on Femtosecond Laser Plasma Shock Wave," Photonic Sensors, 2017, 7(1): 1-10.

\section{Introduction}

In recent years, the material detection methods consist of chemical composition analysis, photo acoustic spectrum analysis, performance test, and so on. Among them, photo acoustic spectroscopy (PAS) is comparatively a new material testing technology. It has made great progress since the 1970s [1]. Rosencwaig and Gersho [2] published one-dimensional photo-acoustic theory for condensed matter in 1976, which is known as the R-G theory. Based on the R-G theory, when using a gas microphone detecting photo-acoustic signal [3], the signal depends on the pressure of gas samples perturbing and disturbing on the interface accepted by the microphone.

The complexity of the Jackson-Ainer theory limits its practical application, and then Blonskij et al. [4] simplified the computing method of piezoelectric photo-acoustic signal. The photo acoustic spectrum detection technology has the advantages of high sensitivity, wide range of detectable spectrum, and non-contact character [5, 6]. It has been applied widely to the detection and analysis for materials. However, the photo acoustic spectrum technology usually adopts radiation light sources including ultraviolet, visible, and infrared laser [7-9], which can produce light saturation phenomenon easily, and it will led to a decrease in the signal-to-noise ratio and influence the effect of detection. The microphone and photo acoustic spectrum test system usually adopts the piezoelectric element (PZT) or uses microphone (MIC) [10]. PZT has the problem of electric charge [11], which would

Received: 6 May 2016 / Revised: 5 September 2016

(C) The Author(s) 2016. This article is published with open access at Springerlink.com

DOI: $10.1007 / \mathrm{s} 13320-016-0340-\mathrm{x}$

Article type: Regular 
affect the sensitivity of system testing. The frequency range for the MIC is quite limited and is affected easily by noise, as we all know that the PAS technology has heating effect.

However, femtosecond laser has the advantage of feeble heating effect which is better in the detecting technology. Therefore, the photo acoustic spectroscopy detection theory remains to be further improved. Based on this, this paper uses optical fiber Fabry-Perot (F-P) acoustic emission sensor probe instead of PZT, which belongs to the fiber external cavity type sensor, and it is suitable for small amplitude signals' detection because of its high sensitivity, with advantages of the anti-interference and sensing probe's smaller type at the same time. It is better than the micro-plasma measurement. Considering the shortcoming of general radiation sources, femtosecond laser has been used as the light source [12-14] in this paper to analyze the change rules of plasma shock wave acoustic emission signal produced by femtosecond laser ablating pure $\mathrm{Cu}, \mathrm{Fe}$, and $\mathrm{Al}$, thus providing a new method for testing materials.

\section{Experiment}

\subsection{Characteristics of the F-P sensor}

(1) The light beam in the F-P sensor is actually located at the center of the quartz diaphragm, and the size of the light spot is far smaller than that of diaphragm. The quartz diaphragm would de deformed under the air pressure $P$, and the maximum deflection is defined as $y$ max. The deflection $y_{\max }$ at the center of a quartz membrane could be considered as the effective deflection, i.e. the amount of actual cavity length change $\Delta d=y_{\max }$. When the signal is detected by the sensor, the diaphragm would oscillate at a very high speed, leading to the quick change in the cavity length. So the interference signal strength $I_{R}$ varies with the cavity length $d$ very fast.

$$
y_{\max }=\frac{3 P\left(1-\mu^{2}\right)}{16 E} \cdot \frac{a^{4}}{h^{3}}
$$

where $h$ stands for the thickness of the diaphragm, $a$ is the effective radius of the diaphragm, $E$ is the Young modulus, and $\mu$ is the Poisson ratio of the diaphragm. The pressure sensitivity of quartz diaphragm at the center can be defined as

$$
s_{\max }=\frac{y_{\max }}{P}=\frac{3\left(1-\mu^{2}\right)}{16 E} \cdot \frac{a^{4}}{h^{3}} .
$$

(2) The modulation method of the optical fiber F-P sensor in the experiment is based on the double beam interference. When the wavelength of the tunable diode laser remains the same, the relation between the reflection intensity $I_{R}$ and the cavity length is a cosine function as

$$
I_{R} \approx I_{0} \cdot 2 R(1-\cos \varphi)=2 R I_{0}\left(1-\cos \frac{4 \pi d}{\lambda}\right)
$$

where $R$ stands for the surface reflectance, $I_{0}$ is the incident light intensity, and they are all constants. $\varphi$ is the phase between two adjacent refracted beams.

(3) The free spectral range (FSR) of the F-P sensor reflects dense degree of the interference fringes. It could be calculated as follows:

$$
\mathrm{FSR}=\left|\lambda_{m 1}-\lambda_{m 2}\right| .
$$

The relation between the length of cavity and FSR could be estimated as

$$
d=\frac{1}{2} \cdot \frac{\lambda_{m 1} \lambda_{m 2}}{\left|\lambda_{m 2}-\lambda_{m 1}\right|} .
$$

The length of cavity could be calculated when knowing $\lambda_{m 1}$ and $\lambda_{m 2}$. From this, we know that the longer the length is, the smaller the FSR is, and the fringes would be more dense.

\subsection{Principles of the detecting method}

The optic signal generated by tunable semiconductor laser would pass into the circulator through the fiber and then into the sensors. The signal would be modulated when the sensor receives the acoustic signal of the plasma shock wave produced by femetosecond ablation targets. Then the modulated signal would be reflected back to the circulator. In this way, the signal owning sensing information is detected by the photoelectric 
conversion and analyzed by the data acquisition system. The modulated information wave could be timely observed in the computer finally. The experimental apparatus is shown in Fig. 1. The experimental femtosecond laser is a kind of femtosecond laser micro-processing system from Japanese Cyber Laser Company, of which the type is titanium gem LS-IF-FW-C-401. The related technology parameters are as follows: the wavelength is $780 \mathrm{~nm}$, the pulse width is $180 \mathrm{fs}$, the average output power is $1.1 \mathrm{~W}$, the pulse frequency is $1 \mathrm{kHz}$, the pulse energy is $1.1 \mathrm{~mJ}$, the pulse stability is $1.5 \% \mathrm{rms}$, the three-dimensional work bench moving range $(x, y, z)$ is $\pm 100 \mathrm{~mm} \times$ $\pm 100 \mathrm{~mm} \times \pm 25 \mathrm{~mm}$, and the workbench mobile precision $(x, y, z)$ is $1.0 \mu \mathrm{m} \times 1.0 \mu \mathrm{m} \times 0.5 \mu \mathrm{m}$. Laser light source is a tunable semiconductor laser light source produced by Santec, of which the model is tunable semiconductor laser TSL-510, with high stability. The wavelength $(\lambda)$ of tunable semiconductor laser light source is $1545 \mathrm{~nm}$, and the power $(P)$ is $0.2 \mathrm{~mW}$ in this experiment. Data acquisition module is a kind of multi-channel data acquisition card made by NI company, with voltage range approximately $0.56 \mathrm{~V}-0.87 \mathrm{~V}, f=600 \mathrm{kHz}$, which ensures the signal without distortion. The photoelectric conversion module is developed independently by our laboratory, composed of photoelectric conversion circuit, signal amplification circuit, and filter circuit. The photoelectric conversion module is a kind of high sensitivity photoelectric signal processor to achieve photoelectric conversion.

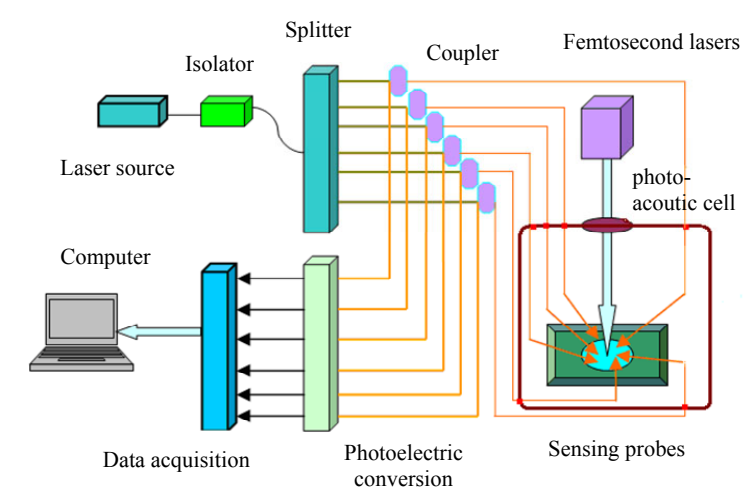

Fig. 1 Experimental device diagram.
In experiments, tunable semiconductor laser has been used as the light source in the measurement process. Femtosecond laser produces femtosecond laser beam with high power density and short pulse, then the beam irradiates pure $\mathrm{Cu}, \mathrm{Fe}$, and $\mathrm{Al}$ target to produce high voltage laser plasma shock wave, using the way of space division multiplexing in a sealed space. The detected signal passes through the coupler and the branching device into the photoelectric signal processing module, then data acquisition module. Finally, we analyze and research the collected signal by computer processing.

As shown in Fig. 2, the sensor has a structure of all-fiber with F-P acoustic emission sensor probe owning an adhesive microstructure. It has characteristics of resistance electromagnetic interference and a wide frequency band. As for the structure of the sensor probe, the capillary glass tube's outside diameter size is identical with the optical fiber. The capillary glass tube is spliced with the optical fiber. The thickness of the capillary glass tube is $50 \mu \mathrm{m}$, and the thin quartz crystal diaphragm is stuck in the surface of capillary glass tube. The quartz crystal diaphragm determines the sensibility of the sensor probe. The diameter of the sensor probe is only $125 \mu \mathrm{m}$. It has high sensitivity and accuracy, and can detect the time distribution and intensity space distribution of femtosecond laser plasma shock wave in the process of formation, development, and attenuation. It realizes accurate measurement of laser plasma shock wave with superiorities such as non-contact, high precision, high response speed, and high sensitivity, which can help us analyze the action mechanism between the femtosecond laser plasma shock wave and different materials.

Figure 3 shows the time domain diagram of the typical femtosecond laser plasma shock wave acoustic emission signal. 


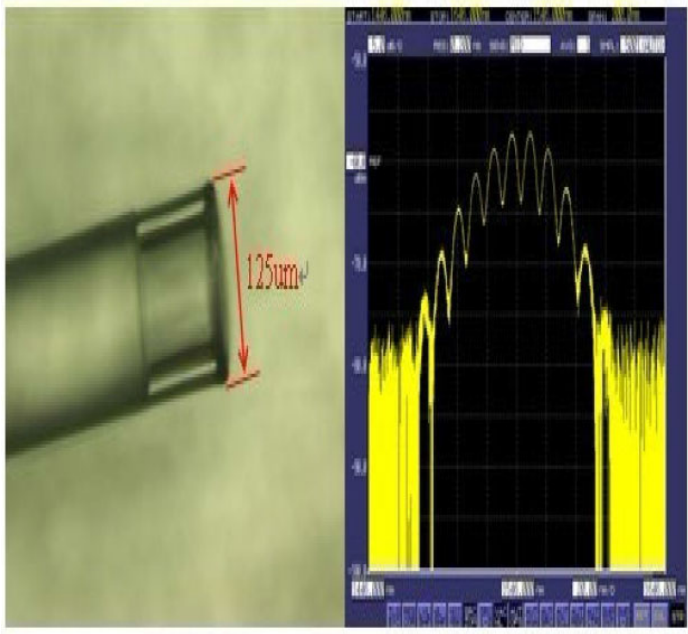

Fig. 2 Optical fiber F-P sensor probe and spectrum.

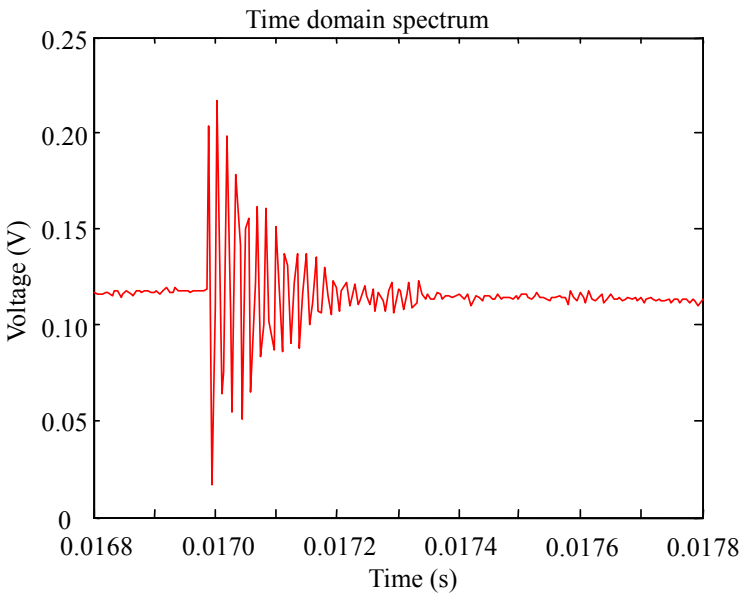

Fig. 3 Acoustic emission signal.

\section{Results and discussion}

To measure and analyze the characteristics of femtosecond laser plasma shock wave acoustic emission signals, we use different laser pulse energies including $300 \mathrm{~mW}, 400 \mathrm{~mW}, 500 \mathrm{~mW}$, $600 \mathrm{~mW}, 700 \mathrm{~mW}$, and $800 \mathrm{~mW}$ with different detection ranges including $1500 \mu \mathrm{m}, 2000 \mu \mathrm{m}$, $3000 \mu \mathrm{m}, 5000 \mu \mathrm{m}, 6000 \mu \mathrm{m}$, and $8000 \mu \mathrm{m}$. What's more, the purity of $\mathrm{Cu}, \mathrm{Fe}$, and $\mathrm{Al}$ is $99.99 \%$. Femtosecond laser ablates the pure $\mathrm{Cu}, \mathrm{Fe}$, and $\mathrm{Al}$ to produce plasma shock wave in a sealed space. Considering that the signal detected by F-P acoustic emission sensor has vibration attenuation which is mainly due to the forced vibration attenuation rules of the sensor piezoelectric membrane, the first peak of the signal frequency can be identified as the laser plasma shock wave acoustic emission signal. We take the first peak as the frequency of acoustic emission signal of the plasma shock wave. The experimental results in Fig. 4 show the change rules of the plasma shock wave acoustic emission signal frequency peak value of pure $\mathrm{Cu}, \mathrm{Fe}$, and $\mathrm{Al}$ under different energies and detection ranges.

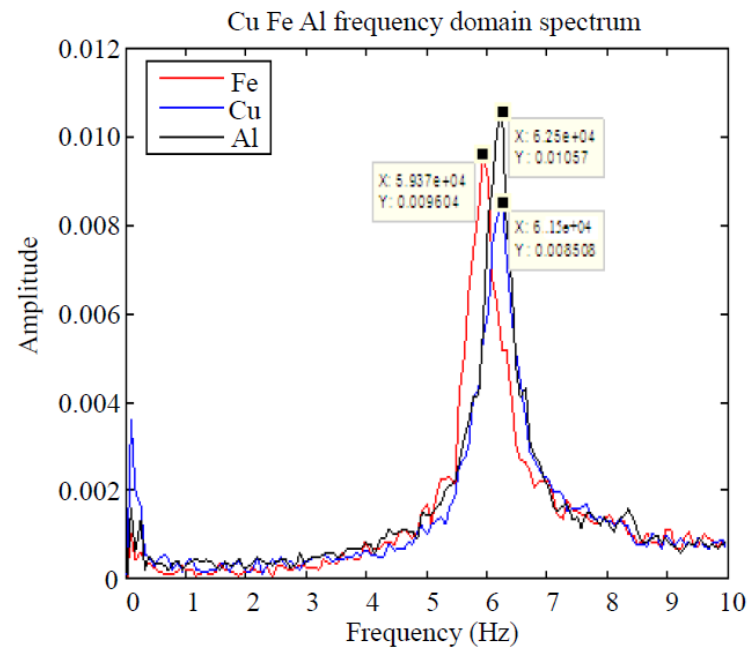

Fig. 4 Frequency change diagrams of pure $\mathrm{Cu}, \mathrm{Fe}$, and $\mathrm{Al}$ plasma shock wave acoustic emission signal with the change pulse energies and detection distances.

From the measurement results of the femtosecond laser ablating the pure $\mathrm{Cu}, \mathrm{Fe}$, and $\mathrm{Al}$ to produce plasma shock wave under changed femtosecond laser pulse energies and detection ranges in the sealed space, we get that the plasma shock wave acoustic emission signal frequency has a certain change rule. In Fig. 4, for the same target material, the plasma shock wave acoustic emission signal frequency remains the same with changed pulse energies and detection ranges. The acoustic emission signal frequency of the femtosecond laser ablating pure $\mathrm{Cu}$ is mainly concentrated in the frequency of $61.5 \mathrm{kHz}$, and the acoustic emission signal frequency of the femtosecond laser ablating pure $\mathrm{Fe}$ and $\mathrm{Al}$ are concentrated in the frequencies of $59.37 \mathrm{kHz}$ and $62.5 \mathrm{kHz}$. Obviously, the acoustic emission signal frequency of the femtosecond laser ablating the pure $\mathrm{Fe}$ is lower than that of the pure $\mathrm{Cu}$ and $\mathrm{Al}$ in the sealed space, what's more it almost remains unchanged. Maede et al. [15] calculated the molten pool's natural oscillation frequency of the 
laser irradiation target, which was below $10 \mathrm{kHz}$. It is visible that the acoustic emission signal of the femtosecond laser plasma shock wave measured in this experiment has a high frequency. In the process of femtosecond laser ablating target material, the strong sound and light signals are similar with lightning. The low frequency features of thunder are related with air expansion rate, for this, we conclude that the detected frequency of the acoustic spectrum is related with the environment gas properties in the sealed space. Furthermore, in the process of interaction between laser and different materials, ionization properties and thermal properties are different. The differences of the doped metal ions and gas vibration intensity of electrons are mainly reflected on the diversities of sound pressures corresponding to different materials under the same experimental condition.

When the femtosecond laser radiation metal target is to meet or exceed the optical breakdown threshold, producing photo-acoustic signal could be expressed as

$$
E_{r}=\xi \chi
$$

where $E_{r}$ is the sound energy of the initial micro plasma, $\chi$ is the threshold energy of laser ablating, and $\xi$ is the photo-acoustic conversion efficiency of pure metal materials. The value of $\xi$ and $\chi$ are constant for the same metal material. The acoustic energy of the initial micro plasma for some kind of metal materials is constant. Therefore, the acoustic signal frequency of the initial plasma for the metal material is also constant. With the expansion of laser micro plasma, the micro plasma acoustic emission signal frequency for the same material should be certain after micro plasma energy attenuation in the same detection distance. These are in good agreement with the results from the experiment.

At the same time, there is the energy coupling of laser and metal in the process of laser acting on metal materials. Under the same laser conditions, the absorbing laser energy is different for the metals of different electrical conductivities. The absorption coefficient (in power) [16] could be expressed as

$$
\begin{gathered}
A(\theta)=\frac{2 \Delta \cos \theta}{\left(\omega_{p} / \omega\right)^{2} \sqrt{\left(\omega_{p} / \omega\right)^{2}-\cos \theta^{2}}} \times \\
{\left[\frac{\left(\omega_{p} / \omega\right)^{2}-\cos 2 \theta}{\left(\omega_{p} / \omega\right)^{2} \cos ^{2} \theta-\cos 2 \theta}\right]} \\
\Delta=\left(\omega \varepsilon_{0} / \sigma\right)\left(\omega_{p} / \omega\right) 4
\end{gathered}
$$

where $\omega_{p}$ is the electron plasma frequency, $\omega$ is the frequency of the laser light, $\varepsilon_{0}$ is the free space permittivity, and $\sigma$ is the electrical conductivity of the metallic target.

For $\Delta<<1$ and $\omega_{p} / \omega>1$, where $\theta$ is the incident angle. As everyone knows, the numerical relationship of the electrical conductivity among these metal materials is $\sigma_{\mathrm{Fe}}>\sigma_{\mathrm{Cu}}>\sigma_{\mathrm{Al}}$. It can derive the numerical relationship of the absorbed laser energy for $\mathrm{Al}, \mathrm{Cu}$, and $\mathrm{Fe}$ that $E_{\mathrm{Al}}>E_{\mathrm{Cu}}>E_{\mathrm{Fe}}$. In addition, the target ablation is related to the first materials ionization. The lower the first ionization energy of target materials becomes, the easier the ablation is, which leads to a bigger micro plasma density. The first ionization energies (EL) for the Al, $\mathrm{Cu}$, and $\mathrm{Fe}$ are different $[\mathrm{EL}(\mathrm{Al})=5.97 \mathrm{eV}$, $\mathrm{EL}(\mathrm{Cu})=7.72 \mathrm{eV}, \mathrm{EL}(\mathrm{Fe})=7.83 \mathrm{eV}]$. Considering the influence of this comprehensive action of the absorbed laser energy and the first ionization energy influence on the target ablation, it could be inferred that the numerical relationship of the micro plasma density for $\mathrm{Al}, \mathrm{Cu}$, and $\mathrm{Fe}$ is $\rho_{\mathrm{Al}}>\rho_{\mathrm{Cu}}>\rho_{\mathrm{Fe}}$. As a result of the expansion and friction with background gas of the different density micro plasma, it would lead to different acoustic emission frequency signals at the same range. Figure 2 shows that the frequency constants for $\mathrm{Al}, \mathrm{Cu}$, and $\mathrm{Fe}$ target are $62.5 \mathrm{kHz}$, $61.5 \mathrm{kHz}$, and $59.37 \mathrm{kHz}$, respectively. It indicates that the greater the micro plasma density is, the higher the frequency of the acoustic emission signal is. It could be inferred that the acoustic emission frequency signal is related to the micro plasma density.

Our further study found that, through the analysis of the spectrum amplitude of plasma shock 
wave acoustic emission signal, $\mathrm{Fe}$ and $\mathrm{Al}$ can be separated. In Fig. 5, the spectrum amplitude of the acoustic emission signal has a variation trend when the femtosecond laser ablates the pure $\mathrm{Fe}$ and $\mathrm{Al}$ under different incident laser pulse energies and different distances.

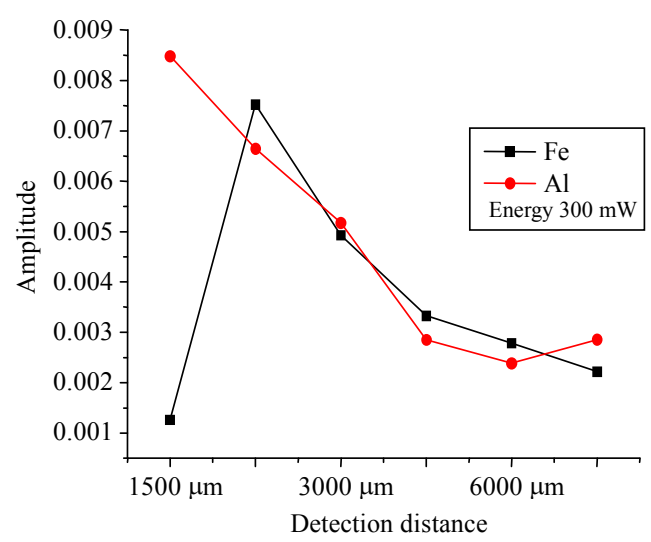

(a)

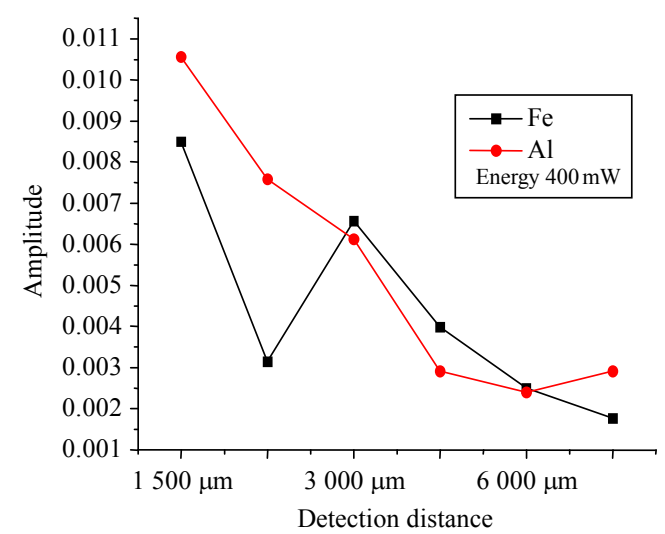

(b)

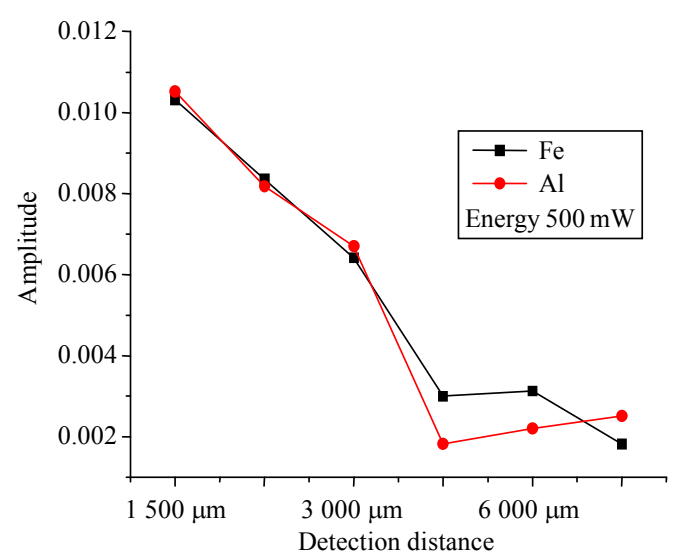

(c)

Fig. 5 Spectrum amplitude of the acoustic emission signal for $\mathrm{Fe}$ and $\mathrm{Al}$ at different energies: (a) energy $300 \mathrm{~mW}$, (b) energy $400 \mathrm{~mW}$, and (c) energy $500 \mathrm{~mW}$.
From Fig. 5, we draw the conclusion that under the same incident laser pulse energy, the spectrum amplitude of the plasma shock wave acoustic emission signal produced by femtosecond laser ablating the pure $\mathrm{Al}$ has a gradually decreasing trend with the change in operating distance. But the spectrum amplitude has a certain fluctuation when ablating the pure $\mathrm{Fe}$. Under different laser pulse energies, we can see that the spectrum amplitude of the pure $\mathrm{Al}$ is higher than that of pure $\mathrm{Fe}$ in the distances of $1500 \mu \mathrm{m}$ and $8000 \mu \mathrm{m}$. So, we can distinguish two kinds of target material of $\mathrm{Fe}$ and $\mathrm{Al}$ according to the ablating characteristics and rules. Through additional experiments, we find that $\mathrm{Cu}$ and $\mathrm{Al}$ have the similar characteristics with $\mathrm{Fe}$ and Al.

From the above analysis, we can use this designed detection system, with an optical fiber F-P acoustic emission sensor probe, and a femtosecond laser as a radiation source to detect the frequency and the spectrum amplitude of the plasma shock wave acoustic emission signal produced by ablating pure $\mathrm{Cu}, \mathrm{Fe}$, and $\mathrm{Al}$, thus determining the composition of the materials. This detection system and method can be applied to the detection of different materials' compositions and characteristics, which provides a new thought for the material test.

In order to further validate test results, the time-frequency signal processing methods have been explored, and the micro plasma shock wave transmit's frequency and energy trace information have been researched in this article. By researching the time-frequency distribution of the micro plasma shock, we can reveal the frequency, energy, and time of duration information. It will be of great importance to explore the femtosecond laser erosion crystalline material dynamics features.

Usually, the micro plasma shock wave acoustic emission signal meets the relation: $x(t) \in L^{2}(R)$, in which the $x(t)$ is limited energy. Meanwhile, we use the tradition Fourier transform method and combine with Parseval, then we can know the signal $x(t)$ in 
the time of total energy and total energy in frequency domain is equal.

$$
E_{x}=\|x(t)\|^{2}=\int_{-\infty}^{\infty}[x(t)]^{2} d t=\frac{1}{2 \pi} \int_{-\infty}^{+\infty}[X(\mathrm{j} \omega)]^{2} d \omega
$$

where $X(\mathrm{j} \omega)$ is the fast Fourier transform (FFT) of $x(t)$. FFT cannot determine different time of the frequency of the details, which can only obtain some basic information of energy distribution in the whole frequency range. It's hard to get the corresponding relation between energy and time. We also know that FFT don't have the ability to automatically adjust the time domain and frequency domain resolution.

In order to break through the limitations of the traditional analysis method, for non-stationary micro plasma shock wave in the acoustic emission signal processing, we put the window function $g(\tau)$ add to short time Fourier transform (STFT). Therefore, the time-frequency analysis can reflect the time-frequency local characteristics.

$$
\begin{aligned}
& \operatorname{STFT}_{x}(t, \omega)=\int x(\tau) g_{t, \omega}^{*}(\tau) e^{-\mathrm{j} \omega \tau} d \tau \\
&=\int x(\tau) g^{*}(t-\tau) e^{-\mathrm{j} \omega \tau} d \tau \\
&=\left\langle x(\tau), g(t-\tau) e^{-\mathrm{j} \omega \tau}\right\rangle . \\
& g_{t, \omega}(\tau)=g_{t, \omega}(\tau-t) e^{\mathrm{j} \omega t}, \quad\left\|g_{t, \omega}(\tau)\right\|=1 .
\end{aligned}
$$

From STFT, it could be comprehended that $x(\tau)$ can be separated by the relevant translation function of $g(\tau)$ on the time axis. We can get the two-dimensional function STFT $x(t, \omega)$ by Fourier transform of these separated signals. In the time domain and frequency domain, the primary function $g(t-\tau) e^{-\mathrm{j} \omega t}$ has the feature of limited support, so we can accomplish to achieve the position information of the time domain by inner product operation for (11). According to the window function $g(\tau)$ when we choose the narrow width $\Delta \tau$ and bandwidth $\Delta v$, the time-frequency localization can be achieved. Based on the basic theory of the uncertainty principle, the minimum values of $\Delta \tau$ and $\Delta v$ cannot be achieved at the same time. Therefore, we can consider the influence of the factors on the signal processing according to the actual signal analysis. Since the signal $x(t)$ uses spectra, the time domain can be expressed as the amount type:

$$
\begin{aligned}
S_{x}(t, \omega) & =\left|\operatorname{STFT}_{x}(t, \omega)\right|^{2} \\
& =\left|\int x(\tau) g^{*}(t-\tau) e^{-\mathrm{j} \omega \tau} d \tau\right|^{2} .
\end{aligned}
$$

Hamming:

$$
g(n)=0.54-0.46 \cos [2 \pi n /(N-1)]
$$

where $N$ is the number of frequency points, and $n$ is the window function points. We want to get time domain energy distribution of acoustic emission signal by using (11), (12), and (13).

According to this method, we get much information through femtosecond laser ablation of copper alloy (90:10), aluminum, and iron copper alloy $(93: 2: 5)$ in the process of experiment. The typical crystal materials produce the groups of micro plasma shock wave acoustic emission signal as a typical signal. Based on STFT, the time-frequency analysis technology of femtosecond laser ablation crystal material micro plasma shock wave produced by the acoustic emission signal is analyzed and studied.

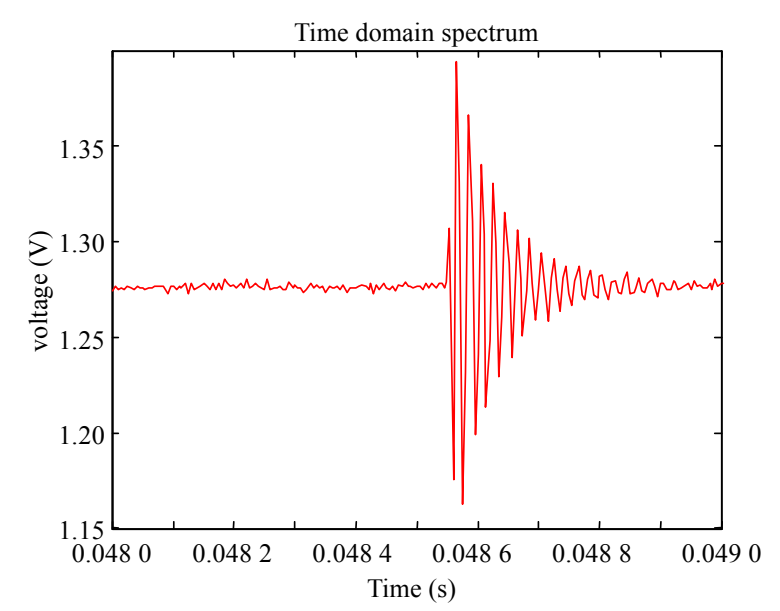

Fig. 6 Typical micro plasma shock wave acoustic emission signal by femtosecond laser ablation of aluminium copper alloy (90:10).

In order to further explore the femtosecond laser ablation characteristics of different crystal materials, 
under the same experimental conditions, the femtosecond laser ablation of aluminum copper alloy micro plasma shock wave produced by the characteristics of acoustic emission signals is studied. Femtosecond laser ablation of aluminum copper alloy (90:10) produced by the micro plasma acoustic emission signal is shown in Fig. 6 .

In the same way, the femtosecond laser ablation of aluminum copper alloy (90:10) produced by acoustic emission signal time-frequency distribution is shown in Fig. 7. The study finds that the aluminum copper alloy (90:10) micro plasma shock wave acoustic emission signals and pure aluminium micro plasma shock wave acoustic emission signal have a similar change rule. But the micro plasma shock wave acoustic emission signal is only one obvious stage, which is in $0 \mathrm{kHz}^{-4} \mathrm{kHz}$ and $40 \mathrm{kHz}^{-}-60 \mathrm{kHz}$ range. We can see that the micro plasma shock wave acoustic emission signal frequency range of aluminum-copper alloy (90:10) is narrower than that of pure aluminum, meanwhile, the two kinds of acoustic emission signals have different energy densities.

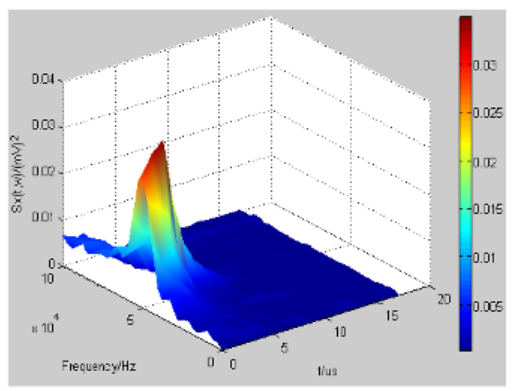

(a)

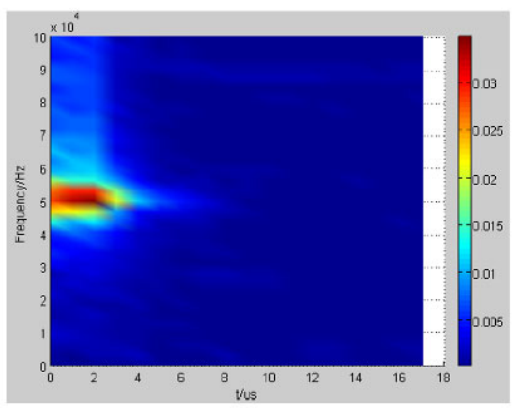

(b)

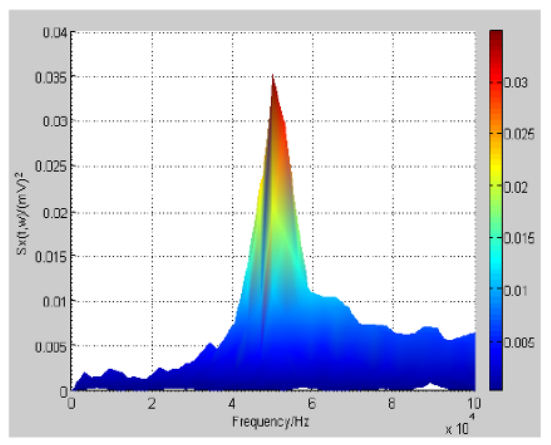

(c)

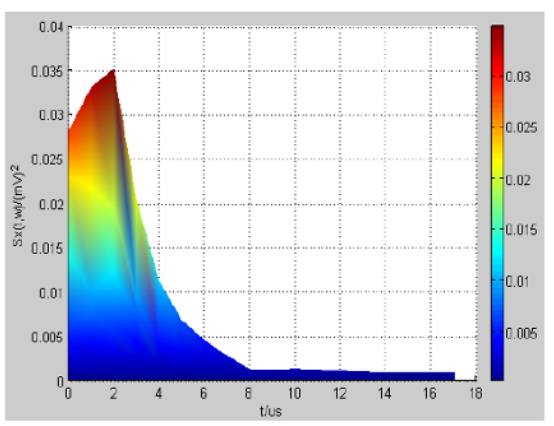

(d)

Fig. 7 Micro plasma shock wave acoustic emission signal time-frequency distribution by femtosecond laser ablation of aluminium copper alloy (90:10): (a) graphic model, (b) plan view $(x-y),(\mathrm{c})$ front view $(x-z)$, and (d) side view $(y-z)$.

In order to explore the characteristics of a wider variety of crystal materials, we have researched the feature of the micro plasma shock acoustic emission signal by femtosecond laser ablating aluminum iron copper alloy (93:2:5) under the same experimental conditions, and the results have been shown in Figs. 8 and 9.

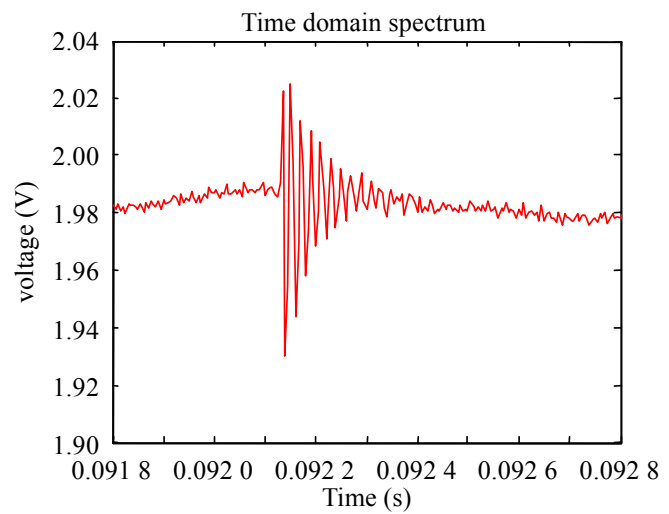

Fig. 8 Typical micro plasma shock wave acoustic emission signal by femtosecond laser ablation of aluminium iron copper alloy $(93: 2: 5)$.

After the same method of micro plasma shock wave acoustic emission signal processing, the result 
has been shown in Fig. 9. Aluminum iron copper alloy micro plasma shock wave acoustic emission signals in $0 \mathrm{kHz}-4 \mathrm{kHz}$ frequency composition is rich, but the frequency range is relatively fuzzy, and we can't get the precise frequency range from picture that the signal distributes the whole area.

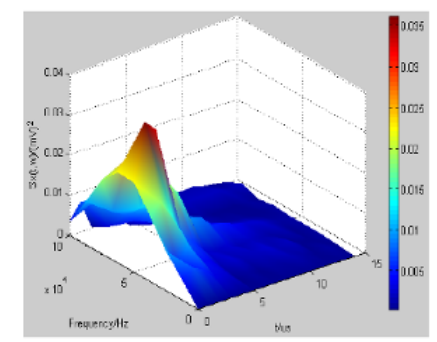

(a)

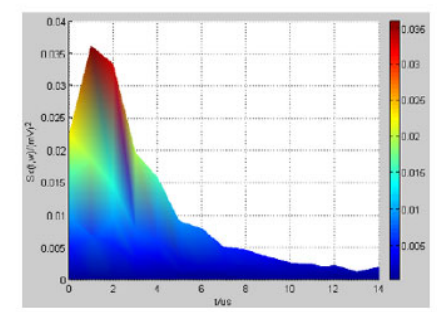

(c)

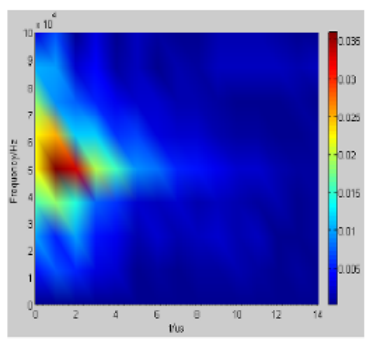

(b)

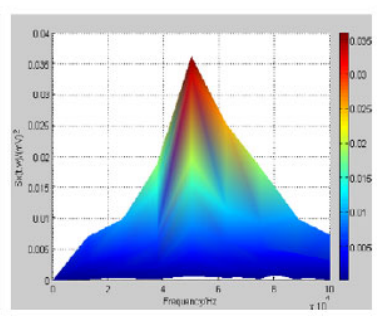

(d)
Fig. 9 Micro plasma shock wave acoustic emission signal time-frequency distribution by femtosecond laser ablation of aluminium iron copper alloy (93:2:5): (a) graphic model, (b) plan view $(x-y),(\mathrm{c})$ front view $(x-z)$, and (d) side view $(y-z)$.

Through time-frequency analysis based on STFT for the femtosecond laser ablating different crystalline materials, we can get the similar characteristics. For example, the spectrum distribution of micro plasma shock wave acoustic emission signal is similar, these signals have close frequency ranges and certain frequency peaks. The different crystal materials have different peak frequencies. We also can conclude that the energy of micro plasma shock wave acoustic emission signal is concentrated in the initial stage. The energy of high frequency component is higher than that of the low frequency component.

From Figs.7 and 9, we can get the STFT time-frequency distribution formula (11), (12), and (13) of the micro plasma shock wave acoustic emission signal $x(t)$.We can research the characteristics of the micro plasma shock acoustic emission signal, which is produced by the femtosecond laser ablation crystal materials. The time-frequency analysis techniques have been used to study micro plasma shock wave acoustic emission signal in the paper, which can provide a new research method for the interaction between the femtosecond laser and material. It will play a guiding role for understanding the interaction mechanism between the femtosecond laser and matter.

\section{Conclusions}

In conclusion, the designed system and the detection method can provide a significant guide for the material testing. For the reason that the interaction mechanism of femtosecond laser and materials is not clear, it needs further research. Ideas and methods of this paper can also provide a further research direction for the interaction between the femtosecond laser and materials.

The research finds that femtosecond laser ablating different crystal materials micro plasma shock wave produced by the acoustic emission signal frequency spectrum distribution of the overall trend is similar. For each kind of crystal materials, under the irradiation of a femtosecond laser energy, producing micro plasma shock wave spectrum distribution of acoustic emission signals remains the same, each kind of materials remains the same, the characteristic frequency of peak signal strength decreases gradually along with an increase in the detection range, the signal intensity increases with an increase in energy, and signal intensity varies for the different crystalline materials.

\section{Acknowledgment}

The authors gratefully acknowledge the financial support for this work provided by the Dr. Start-up Fund of Hubei University of Science and Technology under Grant No. BK1524 and the Science Research Project in Hubei Province 
Department of Education under Grant No. B2015077, the National Natural Science Foundation of China (NSFC) under Grant No. 61575148, the Colleges and Universities of Hubei Province Innovation and Entrepreneurship Training Plan under Grant No. 201510927017, No. 201510927018, and the Teaching Reform Program of Hubei University of Science and Technology under Grant No. 2015-XA-007.

Open Access This article is distributed under the terms of the Creative Commons Attribution 4.0 International License (http://creativecommons.org/licenses/by/4.0/), which permits unrestricted use, distribution, and reproduction in any medium, provided you give appropriate credit to the original author(s) and the source, provide a link to the Creative Commons license, and indicate if changes were made.

\section{References}

[1] S. I. Anisimov, B. L. Kapeliovich, and T. L. Perelman, "Electron emission from metal surfaces exposed to ultrashort laser pules," Zhurnal Eksperimentalnoi I Teroreticheskoi Fiziki, 1974, 66(776): 776-781.

[2] A. Rosencwaig and A. Gersho, "Theory of the photo acoustic effect with solids," Photoacoustic Effect: Vieweg, 1984, 1(6): 631-636.

[3] J. S. Lv and H. Qi. "Multi-wavelength narrow line width fiber laser based on distributed feed back fiber lasers," Photonic Sensors, 2016, 6(3): 256-260.

[4] V. Blonskij, V. A. Thaoryk, and M. L. Shendeleva, "Thermal diffusivity of solids determination by photo acoustic piezoelectric technique," Journal of Applied Physics, 2003, 93(1): 790.

[5] P. Sprangle, J. R. Penano, B. Hafizi, R. F. Hubbard, A. Ting, D. F. Gordon, et al., "Propagation of ultra-short, intense laser pulses in air," Physics of Plasmas, 2004, 11(5): 2865-2874.

[6]J. K. Chen, J. E. Beraun, L. E. Grimes, and D. Y. Tzou, "Modeling of femtosecond laser-induced non-equibrium deformation in metal films,"
International Journal of Solids and Structures, 2002, 39(12): 3199-3216.

[7]W. P. Leemans, B. Nagler, A. J. Gonsalves, C. Toth, K. Nakamura, C. G. R. Geddes, et al., "GeV electron beams from a centimetre-scale accelerator," Nature Physics, 2006, 2(10): 696-699.

[8] S. V. Garnov, V. V. Bukin, A. A. Malyutin, and V. V. Strelkov, "Ultra fast space time and spectrum time resolved diagnostics of multicharged femtosecond laser micro plasma," in AIP Conference Proceedings, Hawaii, vol. 1153, pp. 37-48, 2009.

[9] H. Ditlbacher, J. R. Krenn, G. Schider, A. Leitner, and F. Aussenegg, "Two-dimensional optics with surface plasmon polaritons," Applied Physics Letters, 2002, 81(10): 1762-1764.

[10] D. Zhong and X. Tong. "Application research on hydraulic coke cutting monitoring system based on optical fiber sensing technology," Photonic Sensors, 2014, 4(2): 147-151

[11] A. Y. Basharin, V. S. Dozhdikov, V. T. Dubinchuk, A. V. Kirillin, I. Y. Lysenko, and M. A. Turchaninov, "Phases formed during rapid quenching of liquid carbon," Technical Physics Letters, 2009, 35(5): 428-431.

[12] J. Chen, G. Conache, M. Pistol, S. Gray, M. T. Borgström, H. Xu, et al., "Probing strain in bent semiconductor nanowires with Raman spectroscopy," Nano Letters, 2010, 10(10): 1280-1286.

[13] X. L. Tong, D. S. Jiang, W. B. Hu, Z. M. Liu, and M. Z. Luo, "The comparison between CdS thin films grown on Si (111) substrate and quartz substrate by femtosecond pulsed laser deposition," Applied Physics A, 2006, 84(1-2): 143-148.

[14] M. Beresna, P. G. Kazansky, Y. Svirko, M. Barkauskas, and R. Danielius, "High average power second harmonic generation in air," Applied Physics Letters, 2009, 95(12): 121502-1-121502-3.

[15] T. Maede, E. Ohmura, and I. Miyamoto, "Analysis of key hole behavior in laser welding," in Proceeding of 6th International Symposium of Japan Welding Society, Nagoya, pp. 104-105, 1998.

[16] M. Nikoufard, M. K. Alamouti, and A. Adel, "Ultra-compact photonic crystal based water temperature-sensor," Photonic Sensors, 2016, 6(3): 274-278. 